\title{
Bolometric light detectors for Neutrinoless Double Beta Decay search
}

\section{Margherita TENCONI ${ }^{a b}$ Dmitry CHERNYAK, ${ }^{a b c}$ Fedor DANEVICH, ${ }^{c}$ Andrea GIULIANI, ${ }^{d}$ Michele MANCUSO, ${ }^{a d}$ Stefanos MARNIEROS, ${ }^{a}$ Emiliano OLIVIERI, ${ }^{a}$ Claudia RUSCONI ${ }^{d e}$}

${ }^{a}$ Centre de Spectrometrie Nucleaire et de Spectrometrie de Masse F-91405 Orsay, France

${ }^{b}$ Université Paris-Sud

F-91405 Orsay, France

${ }^{c}$ Institute for Nuclear Research

MSP 0368 Kyiv, Ukraine

${ }^{d}$ Università degli Studi dell'Insubria, Dipartimento di Fisica e Matematica

I-22100 Como, Italy

${ }^{e}$ Istituto Nazionale di Fisica Nucleare, Sezione di Milano-Bicocca

I-20126 Milano, Italy

E-mail: tenconi@csnsm.in2p3. fr

\begin{abstract}
Bolometric light detectors provide an efficient method to collect light signals at small thresholds in cryogenic experiments and are being exploited in present and next-generation experiments for the search of Dark Matter and Neutrinoless Double Beta Decay. In this paper, we discuss the performance of sample light detecting bolometers made up of a ultra-pure Ge slab read by a high impedance thermistor, which were assembled and tested at very low temperatures, below $30 \mathrm{mK}$; we report about signal amplitude, signal to noise ratio, energy resolution and reproducibility. The purpose of this investigation is to develop hybrid detectors to search for Neutrinoless Double Beta Decay.
\end{abstract}

International Workshop on New Photon-detectors,

June 13-15, 2012

LAL Orsay, France

\footnotetext{
* Speaker.
} 


\section{Introduction and goals}

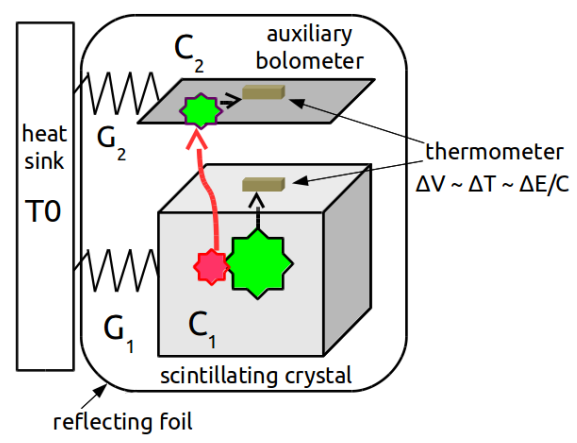

Figure 1: Schematics of a typical scintillating bolometer setup: light detectors are thin-absorber-bolometers, placed in front of the scintillating crystal.
Neutrinoless Double Beta Decay (0vDBD) is a nuclear process playing a key role in particle physics: its observation would be the first proof of lepton number violation, revealing the Majorana nature of the neutrino and providing in addition an evaluation of its effective mass and hierarchy of mass eigenvalues $[1,2,3,4]$. The bolometric technique [5] has already proved to be a powerful method in this field, allowing to build large scale experiments and retaining at the same time high efficiencies and energy resolutions. By means of double read-out of phonon and scintillation signals produced by particle interactions, it is possible to discriminate $\beta$-like events from those induced by $\alpha \mathrm{s}$, the latter being a dangerous source of background in the energy region of interest for many candidate isotopes. This can be accomplished using scintillating bolometers (figure 1), where the photons produced in the main scintillating crystal absorber are collected by a light detector, consisting of another auxiliary bolometer, in the form of a thin slab opaque to the emitted light.

The main purpose of our work is to probe and optimize the light sensor performances, such as sensitivity, energy resolution and reproducibility, in view of the assembly of a next-generation large mass $0 v \mathrm{DBD}$ experiment.

\section{Experimental methods}

Light detectors concerned in this work are made up of an ultrapure Ge absorber, held in a copper frame by PTFE pieces; the temperature sensor is a Ge Neutron Transmutation Doped thermistor (NTD) [6], glued on the absorber's surface with spots of Araldite epoxy glue. Electric and thermal connections are provided by $25 \mu \mathrm{m}$ golden wires. The detectors are cooled down to very low temperatures, in a range from 15 to $30 \mathrm{mK}$, by means of a dilution refrigerator. Calibration is performed by means of a ${ }^{55} \mathrm{Fe}$ source, placed in front of the Ge absorber, emitting X-rays peaking at 5.9 and $6.4 \mathrm{keV}$. In some tests, the Ge absorbers are placed in front of a scintillating crystal, sometimes irradiated with external sources, so as to probe the $\alpha / \beta$ discrimination capability of the whole scintillating bolometer setup and its energy resolution. We considered in our tests $\mathrm{ZnSe}$ and $\mathrm{ZnMoO}_{4}$ scintillating crystals, whose emission spectrum peaks at around $645 \mathrm{~nm}$ and $625 \mathrm{~nm}$ respectively.

Having to perform serial R\&D tests, easy-assembling, reproducibility and adaptability to different detector setups have to be fulfilled: a first, non-negligeable aim of this work was the search for an optimal design for the light detector itself. Different types of setups were tested, changing absorber dimension and thickness, PTFE arrangement, NTD type and size.

The first light detector prototypes were assembled in the cryogenic laboratory of Insubria University, Italy, and consisted of bare square shaped Ge slabs, $15 \mathrm{~mm}$ side and $0.5 \mathrm{~mm}$ thickness, 
equipped with $3 \times 1 \times 0.6 \mathrm{~mm}^{3}$ NTDs. The absorbers are held trasversally by slit PTFE pieces and wires connections are incorporated into the whole detector frame, making it impossible to isolate the light detector without dismounting it completely. A devoted holder was then conceived, in order to be integrated into different setups without compromising the original thermal couplings: Ge absorbers are kept in place by means of PTFE pincers, screwed on an independent copper frame which also hosts pads for wire connections. This kind of setup was adapted to large absorbers as well, $5 \mathrm{~cm}$ diameter and around $250 \mu \mathrm{m}$ thick, equipped with $3 \times 2.2 \times 0.6 \mathrm{~mm}^{3}$ NTDs (figure 4).

\section{Results}

\subsection{Coupling to scintillating crystals}

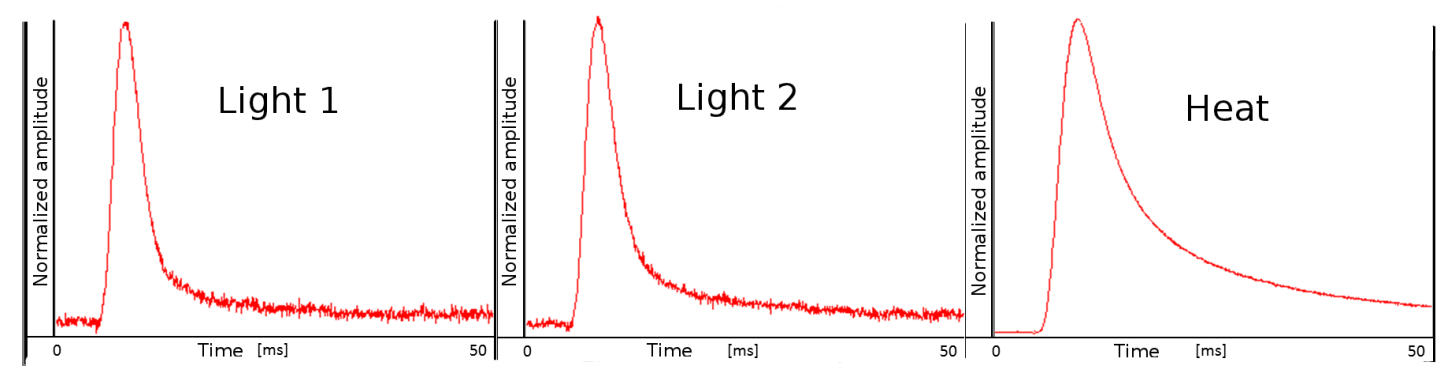

Figure 2: Example of a coincidence event on the $5 \mathrm{~g}$ ZnSe bolometer: registered pulses on the heat channel and the two light detectors facing the scintillating crystal. Signal amplitudes are normalized.

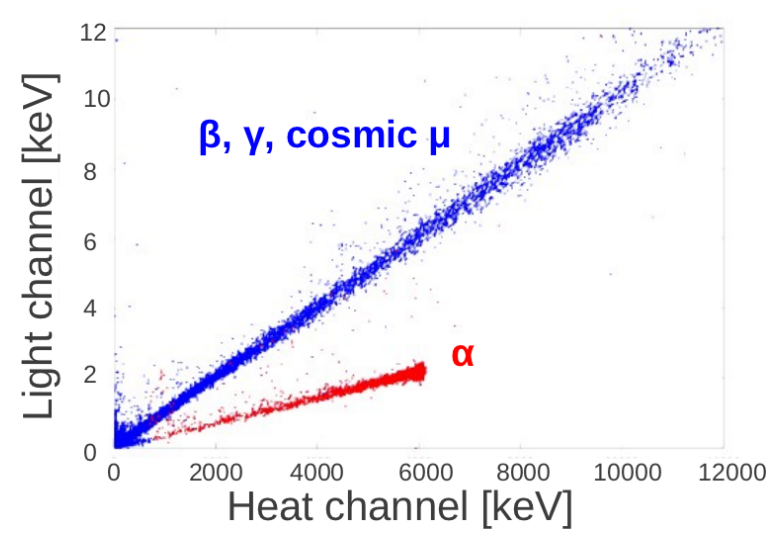

Figure 3: Light yield detected by Ge light detectors as a function of phonon energy deposited in the $5 \mathrm{~g}$ $\mathrm{ZnMoO}_{4}$ scintillating crystal [7]. The run was performed aboveground: high energy portion of the $\beta$ band arises from cosmic muons.

Small light detectors $\left(15 \times 15 \mathrm{~mm}^{2} \mathrm{Ge}\right.$ absorbers), coupled to small $\mathrm{ZnSe}(\sim 5 \mathrm{~g})$ and $\mathrm{ZnMoO}_{4}(\sim 5 \mathrm{~g}$ and $\sim 24 \mathrm{~g})$ crystals, were operated aboveground (University of Insubria - Como and CSNSM - Orsay): our primary goal was to assess light detector performances in a typical luminescent bolometer configuration, in particular $\alpha$ versus $\beta$ discrimination power.

The thermal signal on the main scintillating absorber is of the order of $50 \mathrm{~ms}$, while light detectors generally yield faster signals, due to their smaller heat capacity (figure 2). Coincidence events on the main scintillating crystal (usually referred to as heat channel) and the auxiliary Ge bolometer (light chan$n e l)$ are displayed in the so called scatter plot (figure 3): light channel signal amplitudes as a function of heat channel amplitudes. Two bands are discernible, $\beta$-like events yielding a different amount of light with respect to $\alpha$ s: the quenching factor, the ratio of $\alpha$ to $\beta$ light yield at a fixed deposited energy in the scintillating absorber, is around 5 for $\mathrm{ZnSe}$ and around 0.2 for $\mathrm{ZnMoO}_{4}$. In the energy region of interest, relative $\beta$ events 
light yields range from a few $\mathrm{keVs}$ for $\mathrm{ZnMoO}_{4}$ to tens of $\mathrm{keVs}$ for $\mathrm{ZnSe}$, for $1 \mathrm{MeV}$ deposited energy.

In all these tests, a good separation between $\beta$ and $\alpha$ events was achieved: the two bands can be clearly discriminated in the scatter plot of the coincidences on the main scintillating crystal and the auxiliary bolometer.

\subsection{Reproducibility of large detectors}

A tower of four identical large light detectors was assembled and tested at CSNSM: absorbers are 5 diameter Ge disks, $\sim 250 \mu \mathrm{m}$ thick. This size is likely to be adopted in the construction of a future $0 v \mathrm{DBD}$ experiment, so the run provided a first, important investigation of detectors whose configuration is very close to the final one.

Static characterization showed homogeneous behaviour of the four detectors, symptomatic of similar thermal couplings (figure 4).

The dynamic sensitivities (figure 5), extrapolated from ${ }^{55} \mathrm{Fe}$ and cosmic muon coincidences calibrations, are all compatible as well: detectors signal amplitude is around $1 \mu \mathrm{V}$ for $1 \mathrm{keV}$ de-

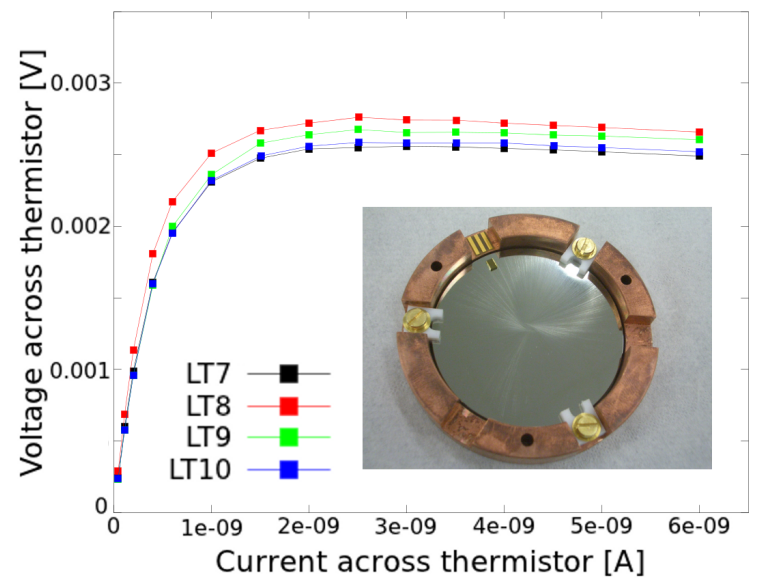

Figure 4: Static characterization of the four large light detectors: voltage across thermistor as a function of polarization current. In the photo, detector setup: $5 \mathrm{~cm}$ diameter ultrapure Ge absorber, $3 \times 2.2 \times 0.6 \mathrm{~mm}^{3} \mathrm{NTD}$ thermistor. posited energy in the Ge crystal, at $19 \mathrm{mK}$ nominal base temperature, while baseline fluctuations vary from $133 \mathrm{eV}$ to $1830 \mathrm{eV}$ due to channel-dependent microphonic noise (figure 6). The worst detector is however still compatible with minimum requirements for its employment in a nextgeneration $0 v$ DBD experiment: in the energy region of interest for ${ }^{100} \mathrm{Mo}$ and ${ }^{82} \mathrm{Se}$, around $3 \mathrm{MeV}$, the collected light is expected to range from a few $\mathrm{keV} / \mathrm{MeV}$ to tens of $\mathrm{keV} / \mathrm{MeV}$, for $\mathrm{ZnMoO}_{4}$ and $\mathrm{ZnSe}$ respectively (see section 3.1). At $17 \mathrm{mK}$ heat sink temperature, one detector showed a baseline noise of $88 \mathrm{eV} \mathrm{FWHM,} \mathrm{the} \mathrm{best} \mathrm{limit} \mathrm{ever} \mathrm{measured} \mathrm{so} \mathrm{far} \mathrm{for} \mathrm{detectors} \mathrm{of} \mathrm{this} \mathrm{type.} \mathrm{A}$ summary of the performed measurements is reported in table 1.

\begin{tabular}{|c|c|c|c|}
\hline Detector & $\mathrm{T}_{b}[\mathrm{mK}]$ & Sensitivity $[\mu \mathrm{V} / \mathrm{keV}]$ & Baseline noise FWHM [eV] \\
\hline LT7 & 19 & 0.79 & 148 \\
\hline LT8 & 19 & 1.02 & 133 \\
& 17 & 1.83 & 88 \\
\hline LT9 & 19 & 0.80 & 184 \\
& 17 & 1.53 & 249 \\
\hline LT10 & 19 & 0.78 & 1830 \\
\hline
\end{tabular}

Table 1: Sensitivities and baseline noise of the four large detectors tested in Orsay. 


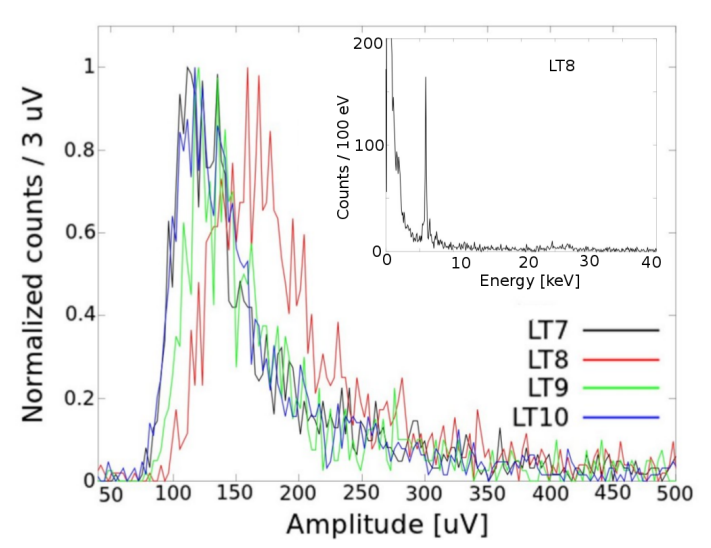

Figure 5: Spectra of muon coincidences in $\mu \mathrm{V}$ : detector responses are comparable. In the inset, a spectrum collected with LT8 shows the ${ }^{55} \mathrm{Fe} 5.9 \mathrm{keV}$ line.

\subsection{Coating effect}

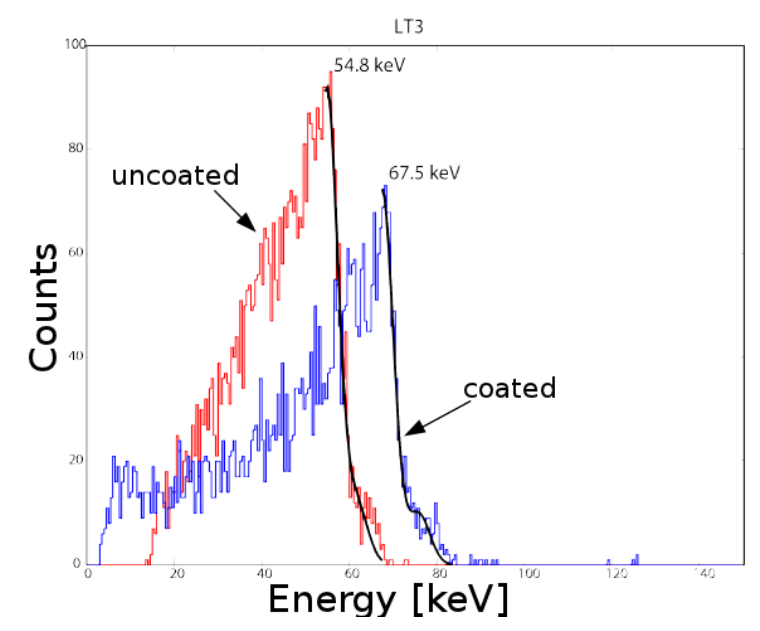

Figure 7: Spectra collected in the two runs with Ge light detectors: without sputtering and coated by $\mathrm{SiO}_{2}$. Results are reproducible and show a gain of the order of $20 \%$ when the surface exposed to the light source is $\mathrm{SiO}_{2}$ coated.

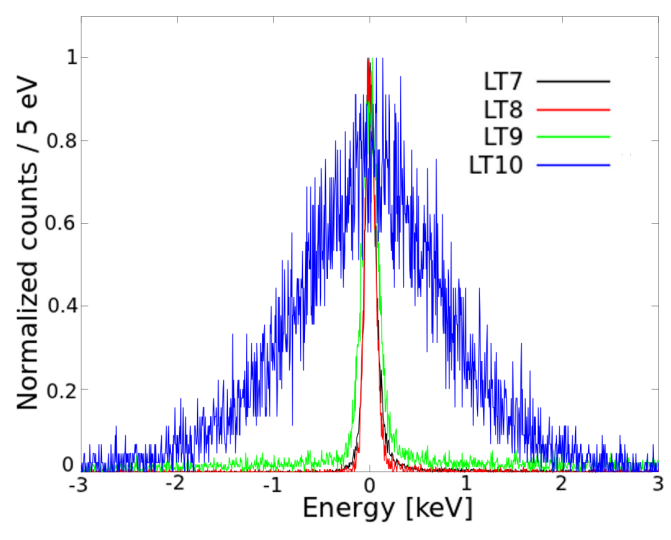

Figure 6: Baseline fluctuation spectra in keV, largely spread due to channel-dependent microphony. The worst FWHM value is however still acceptable in the $0 v \mathrm{DBD}$ scope.

A dedicated setup was constructed in Como cryogenic laboratory in order to study the effect of the deposition of a thin layer of $\mathrm{SiO}_{2}$ by radio frequency (RF) sputtering on the surface of $\mathrm{Ge}$ wafers: $\mathrm{SiO}_{2}$ has a refraction index value intermediate with respect to Ge's and vacuum's, which, according to the index matching anti-reflective method, should increase the absorption efficiency of the coated material with a gain of the order of $22 \%$ with $\mathrm{SiO}_{2}$ in amorphous form.

Four nominally identical light detectors were assembled, the Ge absorber being coated with an approximately $70 \mathrm{~nm}$ thick $\mathrm{SiO}_{2}$ layer only on one side. Each detector was equipped with a heating device [8], directly glued on $\mathrm{Ge}$, in order to periodically dissipate a fixed energy signal into the absorber. Light sources were fabricated by depositing a liquid uranium solution on a thin $\mathrm{ZnSe}$ slab: the expected light spectrum pattern has an end point at the characteristic energy of the source, smearing at lower energies. Two runs were performed, changing the Ge side exposed to the source. The detector responses were compared by referring to heaters pulses and calibrated using minimum ionizing cosmic rays. A gain of the order of $20 \%$ in light collection efficiency was assessed for $\mathrm{SiO}_{2}$ coated sides (figure 7), in reasonable agreement with expectations. 


\section{Conclusions}

Small Ge devices, $15 \mathrm{~mm}$ side, were successfully tested as light detectors for scintillating bolometers. The deposition of a thin $\mathrm{SiO}_{2}$ layer enhances light collection efficiency of about $20 \%$, as a study carried on in Como laboratory showed up. We optimized light detectors design in order for it to suit serial tests and different setups for scintillating bolometers studies. Four nominally identical large Ge devices, $5 \mathrm{~cm}$ diameter, were tested and their good performances were assessed. Static patterns and sensitivities are homogeneous and baseline noise values, though spread due to microphonics, are all within acceptable limits for the detectors to be successfully employed in future $0 v \mathrm{DBD}$ experiments: these detectors will be used in the LUCIFER [9] program and on large $\mathrm{ZnMoO}_{4}$ crystals.

Next step will be the investigation of 5-cm-diameter Ge detectors coupled to a large scintillating crystal: a $313 \mathrm{~g} \mathrm{ZnMoO}_{4}$ bolometer was assembled in CSNSM, Orsay (France) and it will be cooled down at Laboratoire Souterrain de Modane.

\section{Acknowledgments}

The research here described has been performed partially within the project LUCIFER, funded by the European Research Council under the EU Seventh Framework Programme (ERC grant agreement n. 247115), and partially within the program of ISOTTA, a project receiving funds from the ASPERA 2nd Common Call dedicated to R\&D activities.

\section{References}

[1] F.T. Avignone III, S.R. Elliott, J. Engel, Double beta decay, Majorana neutrinos, and neutrino mass, Rev. Mod. Phys. 80 (2008) 481.

[2] A. Giuliani, Search for neutrinoless double beta decay, Acta Phys. Polon. B 41 (2010) 1447.

[3] J.J. Gomez et al., The search for neutrinoless double beta decay, Riv. Nuovo Cim. 35 (2012) 29-98 [hep-ex/1109.5515].

[4] J.D. Vergados, H. Ejiri and F. Simkovic, Theory of neutrinoless double-beta decay, Rep. Prog. Phys. 75 (2012) 106301 [hep-ph/1205.0649].

[5] A. Giuliani, Particle and radiation detection with low-temperature devices, Physica B 280 (2000) 501.

[6] E.E. Haller et al., NTD germanium: a novel material for low temperature bolometers, Proceedings of the Fourth Neutron Transmutation Doping Conference, Neutron Transmutation Doping of Semiconductor Materials, R.D. Larrabee (Ed.), Plenum Press, NY, 1982, pp. 21-36.

[7] J.W. Beeman et al., A next generation neutrinoless double beta decay experiment based on $\mathrm{ZnMoO}_{4}$ scintillating bolometers, Phys. Lett. B 710 (2012) 318 [hep-ex/1112 . 3672].

[8] E. Andreotti et al., Production, characterization, and selection of the heating elements for the response stabilization of the CUORE bolometers, Nucl. Instrum. Methods A 664 (2012), 161.

[9] A. Giuliani et al., LUCIFER: an experimental breakthrough in the search for neutrinoless double beta decay, proceedings of the 5th International BEYOND 2010 Conference, Cape Town, South Africa (2010), World Scientific. 